\title{
Large dust grains in the inner region of circumstellar disks
}

\author{
A. Isella ${ }^{1,2}$, L. Testi ${ }^{1}$, and A. Natta ${ }^{1}$ \\ 1 Osservatorio Astrofisico di Arcetri, INAF, Largo E.Fermi 5, 50125 Firenze, Italy \\ e-mail: isella@arcetri.astro.it \\ 2 Dipartimento di Fisica, Universitá di Milano, via Celoria 16, 20133 Milano, Italy
}

Received 5 December 2005 / Accepted 19 January 2006

\begin{abstract}
Context. Simple geometrical ring models account well for near-infrared interferometric observations of dusty disks surrounding pre-main sequence stars of intermediate mass. Such models demonstrate that the dust distribution in these disks has an inner hole and puffed-up inner edge consistent with theoretical expectations.

Aims. In this paper, we reanalyze the available interferometric observations of six intermediate mass pre-main sequence stars (CQ Tau, VV Ser, MWC 480, MWC 758, V1295 Aql and AB Aur) in the framework of a more detailed physical model of the inner region of the dusty disk. Our aim is to verify whether the model will allow us to constrain the disk and dust properties.

Methods. Observed visibilities from the literature are compared with theoretical visibilities from our model. With the assumption that silicates are the most refractory dust species, our model computes self-consistently the shape and emission of the inner edge of the dusty disk (and hence its visibilities for given interferometer configurations). The only free parameters in our model are the inner disk orientation and the size of the dust grains.

Results. In all objects with the exception of $\mathrm{AB}$ Aur, our self-consistent models reproduce both the interferometric results and the near-infrared spectral energy distribution. In four cases, grains larger than $\sim 1.2 \mu \mathrm{m}$, and possibly much larger are either required by or consistent with the observations. The inclination of the inner disk is found to be always larger than $\sim 30^{\circ}$, and in at least two objects much larger.
\end{abstract}

Key words. stars: planetary systems: photoplanetary disks - stars: pre-main sequence - infrared: stars - techniques: interferometric methods: data analysis

\section{Introduction}

Understanding the properties and evolution of the dust grains contained in proto-planetary disks around pre-main sequence stars is important because they are the seeds from which planets may form. We have now strong evidence that grains in disks are very different from the grains in the diffuse interstellar medium and in the molecular clouds from which disks form, as reviewed, e.g., by Natta et al. (2006). In many objects, observations with millimeter interferometers have provided strong evidence that the grains in the outer and cooler regions of the disk (further than $50 \mathrm{AU}$ from the star) have been hugely processed, and have grown from sub-micron sizes to millimeter and centimeter ones. Closer to the star, however, in the regions were planets are more likely to form, observational evidence has been confined to grains close to the disk surface. For these, which however account for a tiny fraction of the total dust mass, emission in the silicate features has shown a correlation between the shape of the feature and its strength that is interpreted as due to growth of the grains from size $a \sim 0.1 \mu \mathrm{m}$ to $a \sim 1 \mu \mathrm{m}$ (van Boekel et al. 2003, 2004; Meeus et al. 2003). In this inner disk, the properties of the grains in the disk midplane are still unknown.

In the last few years, due to the new long baseline nearinfrared interferometers, many important steps forward in the study of the internal regions of circumstellar disks have occurred. The available near-infrared interferometric observations of T Tauri (TTS) and Herbig Ae (HAe) stars (Eisner et al. 2003, 2004; Millan-Gabet et al. 2001; Tuthill et al. 2001;
Monnier et al. 2005) confirm the idea that the inner disk properties are controlled by the dust evaporation process which produce a "puffed-up" inner rim at the dust destruction radius (Natta et al. 2001; Dullemond et al. 2001, hereafter DDN01). In these models, the location and shape of the rim depends on the properties of grains located not on the disk surface but on its midplane.

Isella \& Natta (2005, hereafter IN05) have recently proposed models of the "puffed-up"inner rim which include a selfconsistent description of the grain evaporation and its dependence on the gas density. IN05 have explored a large range of grain properties, and discussed how the location of the rim depends on grain properties. In this paper, we will use the IN05 models to analyze the existing interferometric data of the best observed HAe stars to explore, in practice, the constraints on grain properties provided by this technique and their uncertainties.

As a byproduct of the modeling process, one obtains also the orientation of the inner disk (i.e. its inclination with respect to the line of sight and its position angle); this can be compared with the orientation of the outer disk, obtained from millimeter observations of the molecular gas and dust emission and/or scattered light in the optical.

The paper is organized as follows. In Sect. 2 we describe the available interferometric observations of the target stars. The IN05 model for the inner rim is briefly summarized in Sect. 3 and used to fit the observations of the individual objects in Sect. 4. A comparison of the results with previous analysis of the same 
Table 1. Stellar parameters.

\begin{tabular}{ccccclll}
\hline \hline Source & Alternate name & Spectral type & $\begin{array}{c}d \\
(\mathrm{pc})\end{array}$ & $\begin{array}{l}T \\
(\mathrm{~K})\end{array}$ & $\begin{array}{l}L \\
\left(L_{\odot}\right)\end{array}$ & $\begin{array}{l}M \\
\left(M_{\odot}\right)\end{array}$ & $A_{\mathrm{v}}$ \\
\hline AB Aur & HD 31293 & A0pe & 144 & 9772 & 47 & 2.4 & 0.5 \\
MWC 480 & HD 31648 & A2/3ep+sh & 140 & 8700 & 25 & 2.2 & 0.25 \\
MWC 758 & HD 36112 & A5IVe & 230 & 8128 & 22 & 2.0 & 0.22 \\
CQ Tau & HD 36910 & A8 V/F2 IVea & 100 & 8000 & 5 & 1.5 & 1.00 \\
VV Ser & HBC 282 & B9/A0 Vevp & 260 & 10200 & 49 & 3.0 & 3.6 \\
V1295 Aq1 & HD 190073 & B9/A0 Vp+sh & 290 & 8912 & 83 & 4.3 & 0.19 \\
\hline
\end{tabular}

Stellar parameters are from Hillenbrand et al. (1992), van den Ancker et al. (1998), Chiang et al. (2001), Strizys et al. (1996), Mannings et al. (2000) and references therein.

data is presented in Sect. 5. Our results are discussed in Sect. 6. Conclusions follow in Sect. 7.

\section{Target stars and observations}

Our sample is composed of six HAe stars (AB Aur, CQ Tau, VV Ser, MWC 480, MWC 758 and V1295 Aql), for which near-infrared interferometric observations exist in the literature. Table 1 summarizes the physical properties of the target stars. All the stars are classified as young stellar objects with masses ranging from 1.5 to 4.3 solar mass and a spectral type between A0/B9 and A8/F2. CQ Tau and VV Ser belong to the family of UXORs and are characterized by large and irregular variability.

We use visibility measurements of the target stars from the literature, obtained with interferometric observations carried out with PTI (Palomar Testbed Interferometer) in $K$ band $\left(\lambda_{0}=2.2 \mu \mathrm{m}, \Delta \lambda=0.4 \mu \mathrm{m}\right)$ described in Eisner et al. (2004). For AB Aur and V1295Aql, IOTA observations are also available (Millan-Gabet et al. 2001) for the $K^{\prime}\left(\lambda_{0}=2.16 \mu \mathrm{m}\right.$, $\Delta \lambda=0.32 \mu \mathrm{m})$ and $H\left(\lambda_{0}=1.65 \mu \mathrm{m}, \Delta \lambda=0.30 \mu \mathrm{m}\right)$ bands.

\section{Model description}

We use a model based on the assumption that the near-infrared emission of HAe stars originates in the "puffed up" inner rim which forms in the circumstellar disk at the dust evaporation radius (Natta et al. 2001; DDN01).

In IN05 we revised the concept of the "puffed up" inner rim, introducing the dependence of the dust evaporation temperature on the local gas density, following the dust model of Pollack et al. (1994). The main result is that the surface of the rim presents a curved shape (see Fig. 1), whose features are summarized in the following.

\subsection{The dust evaporation and the "puffed-up" inner rim}

Following the suggestion of Natta et al. (2001), we assume that the dust component of a circumstellar accreting disk is internally truncated by the dust evaporation process, forming an inner hole of radius $R_{\text {evp }}$ inside of which only gas can survive. If the radiation absorption due to this inner gas is negligible (as is often the case; see, e.g., Muzerolle et al. 2004), dust evaporation occurs where the equilibrium temperature $T_{\mathrm{d}}$ of grains embedded in the unattenuated stellar radiation field, equals their evaporation temperature $T_{\text {evp }}$. In IN05 we used an analytical solution of the radiation transfer problem (Calvet et al. 1991, 1992) to calculate the grain temperature inside the disk and we shown that evaporation occurs at a distance from the star that can be expressed as:

$R_{\text {evp }}[\mathrm{AU}]=0.034 \cdot\left(\frac{1500}{T_{\text {evp }}}\right)^{2} \sqrt{\frac{L_{\star}}{L_{\odot}}\left(2+\frac{1}{\epsilon}\right)}$,

where $L_{\star}$ is the stellar luminosity and $\epsilon$ is the ratio of the Planck mean opacity at $T_{\text {evp }}$ to that at the stellar effective temperature $T_{\star}, \epsilon=\kappa_{P}\left(T_{\text {evp }}\right) / \kappa_{P}\left(T_{\star}\right)$. The quantity $\epsilon$ measures the cooling efficiency of the grains; it depends on the wavelength dependence of the absorption efficiency of the grains and varies with grain composition and size.

If the dust in the proto-planetary disk is composed of different types of grains, the location and structure of the inner rim depends on the properties of the grains with the highest evaporation temperature. In the dust model proposed by Pollack et al. (1994), the most refractory grains are silicates for which $T_{\text {evp }}$ is given by the relation

$T_{\text {evp }}(r, z)=2000 \cdot\left[\rho_{\mathrm{g}}(r, z)\right]^{0.0195}$,

valid for the gas density $\rho_{\mathrm{g}}$ in the range between $10^{-18} \mathrm{~g} \mathrm{~cm}^{-3}$ and $10^{-5} \mathrm{~g} \mathrm{~cm}^{-3}$. In the following analysis, we will therefore assume that the inner disk dust is made of silicates, with optical properties given by Weingartner \& Draine (2001); thus, $\epsilon$ is uniquely defined by the grain radius $a$, and we will use $a$, rather than $\epsilon$, as a model parameter.

Assuming that the proto-planetary disk is in hydrostatic equilibrium in the gravitational field of the central star and that it is isothermal in the vertical direction $z$, the gas density $\rho_{\mathrm{g}}(r, z)$ has its maximum value on the midplane and decreases with $z$ as

$\rho_{\mathrm{g}}(r, z)=\rho_{\mathrm{g}, 0}(r) \exp \left(-z^{2} / 2 h(r)^{2}\right)$,

where $h$ is the pressure scale height of the disk. The midplane density can be expressed as a power-law of $r \rho_{\mathrm{g}, 0}(r)=$ $\rho_{\mathrm{g}, 0}\left(r_{0}\right)\left(r_{0} / r\right)^{\gamma}$, with $\gamma$ of the order of 2-3 (see, e.g., Chiang \& Goldreich 1997).

The decrease of $\rho_{\mathrm{g}}$ with $z$, combined with Eq. (2), implies that the silicate evaporation temperature varies from, i.e., $\sim 1500 \mathrm{~K}$ on the midplane (assuming a typical gas density of $\sim 10^{-7} \mathrm{~g} / \mathrm{cm}^{3}$ ) to $\sim 1000 \mathrm{~K}$ at $z / h=6.4$ and $\sim 800 \mathrm{~K}$ at $z / h=8$. Since $T_{\text {evp }}$ decreases with $z$, it is immediately clear from Eq. (1) that the distance from the star at which dust evaporates increases with $z$, describing a curved surface as shown in Fig. 1.

The dependence of $T_{\text {evp }}$ on the gas density is an important factor when computing the shape of the rim in the vertical direction, where the gas density varies by many orders of magnitude while the distance from the star is practically unchanged. In the radial direction, we expect relatively small variations of $\rho_{\mathrm{g}, 0}$, for any reasonable value of the disk mass, so that the distance of 


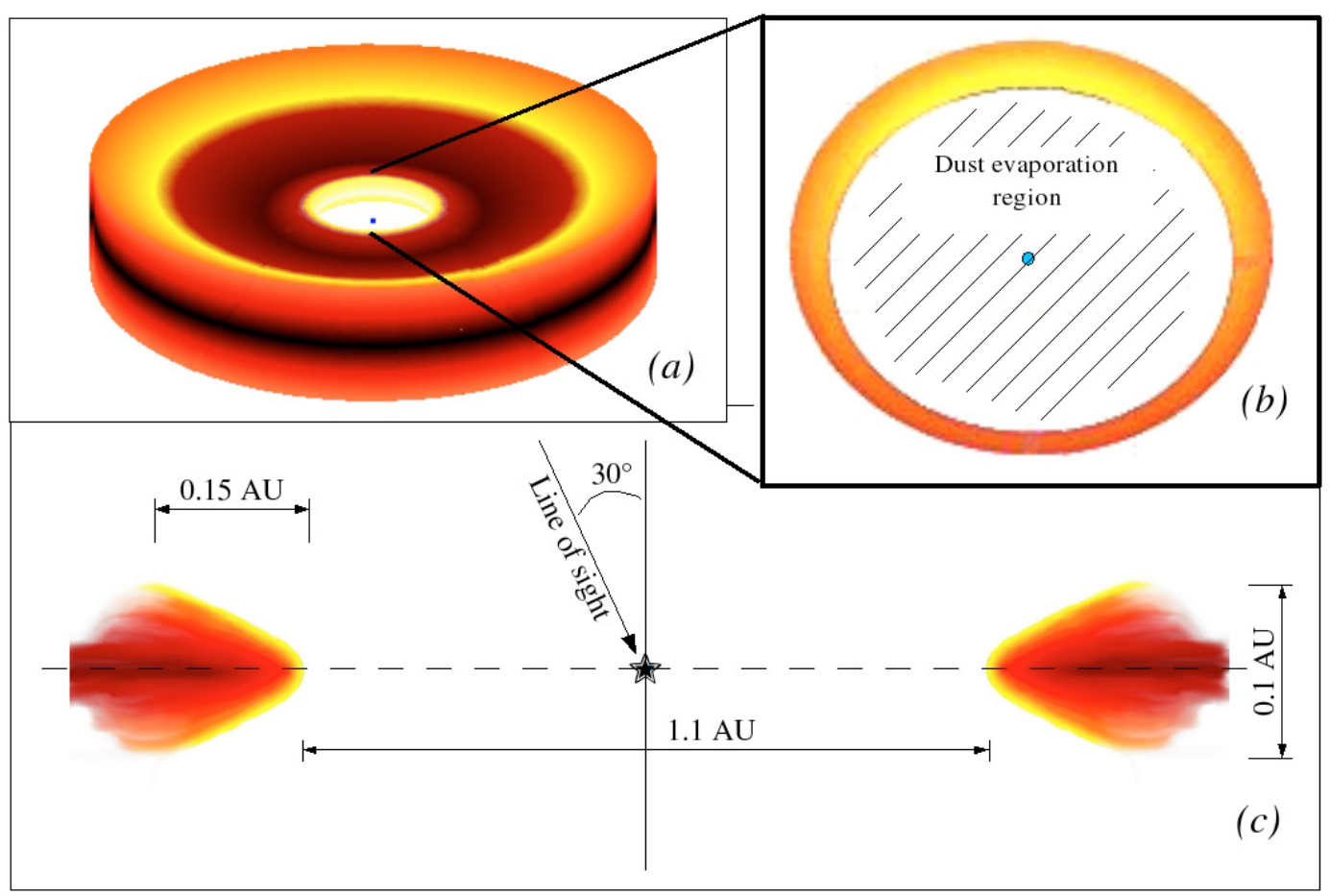

Fig. 1. Sketch of the structure of the inner part of a proto-planetary disk. Panel a) shows a disk with an inner puffed up rim that casts a shadow over the outer part of the disk. Farther than 5-10 AU from the central star, the flaring disk emerges from the rim shadow. Panel b) presents the image of the inner rim, computed with the IN05 models for a star with an effective temperature of $10000 \mathrm{~K}$ and disk inclination of $30^{\circ}$. Panel c) shows a vertical (edge on) section of the inner rim. The curvature of the surface of the rim is caused by the variation of the dust evaporation temperature with the height above the disk midplane (see text).

the rim from the star, measured in the midplane, is practically independent of the gas density.

The emission of the rim is computed assuming that it originates from the surface characterized by an effective temperature $T_{\mathrm{eff}}=T\left(\tau_{\mathrm{d}}=2 / 3\right)$, where $\tau_{\mathrm{d}}$ is the optical depth for the emitted radiation. The $T_{\text {eff }}$ surface, therefore, defines the observed location and shape of the rim. In IN05, we discussed how the $T_{\text {evp }}$ and the $T_{\text {eff }}$ surfaces behave for small and large silicate grains, and showed that the $T_{\text {eff }}$ surface moves closer to the star for increasing grain size until a critical value, which for silicates is about $1.2 \mu \mathrm{m}$. Larger grains produce rims with $T_{\text {eff }}$ surfaces practically independent of $a$. Therefore, for a fixed stellar luminosity, silicates with $a \sim 1.2 \mu \mathrm{m}$ give the minimum value of the distance of the rim from the star. Conversely, if the measured rim distance is equal to this minimum value, one can derive from it only a lower limit $(\sim 1.2 \mu \mathrm{m})$ to the grain size.

The rim emission peaks at near-infrared wavelengths. At $\lambda \lesssim$ 5-7 $\mu \mathrm{m}$, one can assume that the observed flux is the sum of the stellar + rim emission, with only negligible contribution from the outer disk (see, e.g., DDN01). We model the stellar photospheric flux using standard Kurucz model atmospheres.

\subsection{Visibility model}

Due to the limited coverage of the $u-v$ plane of the existing nearinfrared interferometers, it is not possible at present to recover full images from the available data, and one has to resort to the analysis of the visibilities on given interferometric baselines.

Starting from the synthetic images of the inner rim (see IN05), we compute the predicted visibility values using a Fast Fourier Transform recipe. For face-on inclination, due to the circular symmetry of the rim image, the visibility depends only on the length of the baseline $B$. For inclination greater than zero, the image of the rim has an "elliptical" shape: the minor axis decreases with increasing inclination and the upper half of the rim becomes brighter than the lower part. For baselines oriented along the direction of the minor axis of the rim image, the visibility decreases more slowly than for those oriented along the major axis. For all other orientations of the baseline, the visibility will have values intermediate between these two (see Fig. 2). Moreover, due to the Earth rotation during the observation, the baseline corresponding to a fixed telescope pair moves in the $u-v$ plane describing an ellipse. Along this ellipse, each point is related to the hour angle $H A$ of the target object in the sky. In the next section we use the $V^{2}-B$ plot and $V^{2}-H A$ plot, to show how the models fit the observations.

The visibility model takes into account the emission of the central star, modeled as a uniform disk of radius $R_{\star}$. If $F_{\star}$ and $F_{\mathrm{d}}$ are respectively the stellar and the inner rim flux at the wavelength of the observation, the total visibility is given by the relation:

$V^{2}=\left(\frac{F_{\star} V_{\star}+F_{\mathrm{d}} V_{\mathrm{d}}}{F_{\star}+F_{\mathrm{d}}}\right)^{2}$,

where $V_{\star}$ and $V_{\mathrm{d}}$ are the visibility values of the star and of the disk. Note that for the PTI configuration (baselines between $84 \mathrm{~m}$ and $100 \mathrm{~m}$ ) $V_{\star}$ is in all cases very closed to 1 .

\section{Comparison with the observations}

\subsection{Model parameters}

Once the stellar and dust properties are fixed, the modelpredicted visibilities depend on the dust grain radius $a$, which 

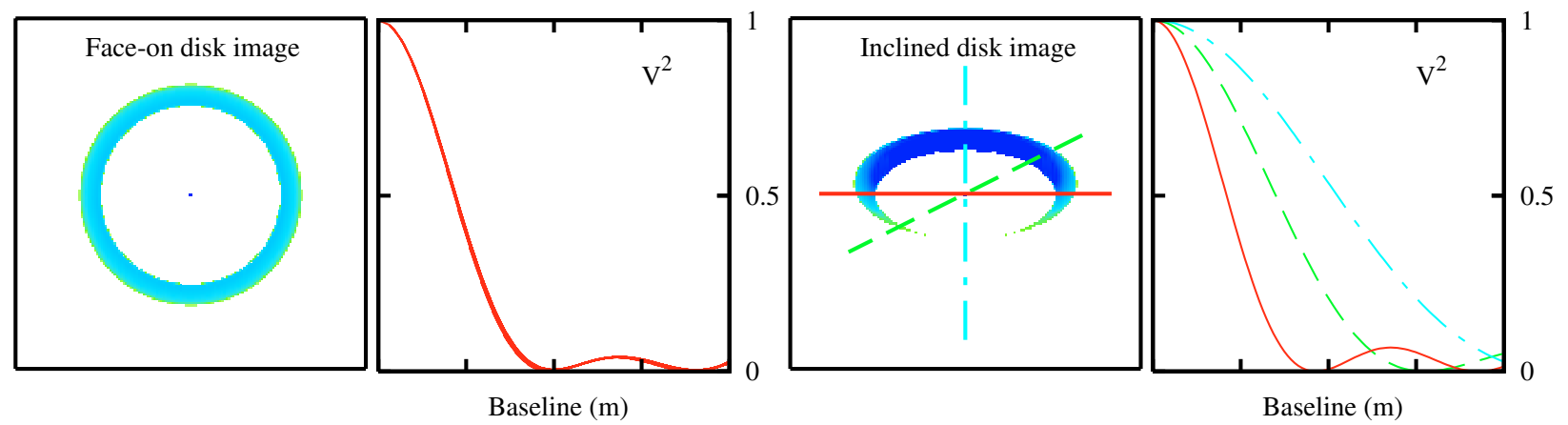

Fig. 2. Predicted visibilities for two different inclinations of the inner rim. The two panels on the left show the predicted image for a face-on rim $\left(\iota=0^{\circ}\right)$ and the relative visibility squared $V^{2}$ : since the image is circularly symmetric, the visibility squared values are the same for every baseline orientation. The two panels on the right show the predicted image for an inclined rim $\left(\iota=60^{\circ}\right)$. In this case, the values of $V^{2}$ depend on the baseline orientations: $V^{2}$ decreases rapidly for baselines oriented along the major axis of the image (solid line) while $V^{2}$ decreases slowly for a baseline oriented along the minor axis (large and small dashes). For the intermediate orientation (dashed line), $V^{2}$ is in between the two extreme values.

Table 2. Best fitting model parameters.

\begin{tabular}{l|llll|lll|ll}
\hline \hline \multirow{2}{*}{ Source } & \multicolumn{4}{|c}{ IN05 model } & \multicolumn{4}{c}{ Eisner et al. (2004) } & \multicolumn{3}{c}{ outer disk } \\
& $\boldsymbol{a}$ & $R_{\text {rim }}$ & $\boldsymbol{\imath}$ & $\mathbf{P A}$ & $R_{\text {rim }}$ & $\iota$ & PA & $\iota$ & PA \\
& $(\mu \mathrm{m})$ & $(\mathrm{AU})$ & $(\mathrm{deg})$ & $(\mathrm{deg})$ & $(\mathrm{AU})$ & $(\mathrm{deg})$ & $(\mathrm{deg})$ & $(\mathrm{deg})$ & $(\mathrm{deg})$ \\
\hline MWC 758 & $\geq \mathbf{1 . 2}$ & 0.32 & $\mathbf{4 0}$ & $\mathbf{1 4 5}$ & 0.21 & $36_{-2}^{+3}$ & $127_{-3}^{+4}$ & 46 & $116_{-5}^{+6 a}$ \\
VV Ser & $\geq \mathbf{1 . 2}$ & 0.54 & $\mathbf{5 0 - 7 0}$ & $\mathbf{6 0 - 1 2 0}$ & 0.47 & $42_{-2}^{+6}$ & $166_{-6}^{+17}$ & $72 \pm 5$ & $13 \pm 5^{b}$ \\
CQ Tau & $\mathbf{0 . 3}-\geq \mathbf{1 . 2}$ & $0.16-0.25$ & $\mathbf{4 0 - 5 5}$ & $\mathbf{1 4 5}-\mathbf{1 9 0}$ & 0.23 & $48_{-4}^{+3}$ & $106_{-5}^{+4}$ & $63_{-15}^{+10}$ & $2 \pm 13^{c}$ \\
V1295 Aql & $\mathbf{0 . 3}-\geq \mathbf{1 . 2}$ & $0.7-1.2$ & $\mathbf{4 0 - 6 5}$ & & 0.55 & $23_{-23}^{+15}$ & & & \\
MWC 480 & $\mathbf{0 . 2 - 0 . 3}$ & $0.53-0.63$ & $\mathbf{3 0}-\mathbf{6 5}$ & & 0.23 & $28_{-1}^{+2}$ & $145_{-6}^{+9}$ & $20-40$ & $147-180^{a, d}$ \\
AB Aur & \multicolumn{3}{|c|}{ impossible to fit } & & 0.25 & $8_{-8}^{+7}$ & & $15-35$ & $50-110^{e, f, g, h}$ \\
\hline
\end{tabular}

From Cols. 2 to 5 are reported the best fit parameters for the "puffed-up" inner rim, obtained with the IN05 model: the grain radius $a$, the radius of the inner rim $R_{\text {rim }}$, the inclination $\iota$ and the position angle PA. The free parameters of the model are presented in bold face. Columns 6-8 show the values of the radius of the inner rim, the inclination and the position angle, obtained by Eisner et al. (2004). Finally, the last two columns show the available estimates of inclination and position angle for the external region of the disk: ${ }^{a}$ Mannings et al. (1997); ${ }^{b}$ Pontoppidan et al. (2006); ${ }^{c}$ Testi et al. (2001, 2003); ${ }^{d}$ Simon et al. (2000); ${ }^{e}$ Fukagawa et al.(2004); ${ }^{f}$ Grady et al. (1999); ${ }^{g}$ Corder et al. 2005; ${ }^{h}$ Piétu et al. (2005).

completely defines the rim structure, and two parameters (observational parameters in the following) that describe the orientation of the disk, namely the inclination $\iota$ and position angle PA.

For each star, we firstly compute the predicted rim structure varying the size of the grains from very small to very large values. As discussed in Sect. 3, we assume that silicates are the most refractory component; we take the optical properties of astronomical silicates defined by Weingartner \& Draine (2001). Other disk parameters (i.e., mass and density radial profile) can be neglected in this analysis. We fix $\rho_{\mathrm{g}, 0}\left(R_{\text {rim }}\right) \sim 10^{-7} \mathrm{~g} / \mathrm{cm}^{3}$ which gives a total disk mass of about $0.1 M_{\odot}$, for a fiducial value of $\gamma=2.5$ and an outer disk radius of the disk of $200 \mathrm{AU}$.

Once the structure of the "puffed up" inner rim is calculated, the predicted visibility depends on the orientation of the disk in the sky, defined by the inclination $\iota$ of the midplane of the disk with respect to the line of sight and its position angle PA, measured from north to east and relative to the major axis of the projected image of the disk on the sky. The inclination is defined so that $\iota=0^{\circ}$ identifies a face-on disk while $\iota=90^{\circ}$ corresponds to an edge-on disk. For inclinations higher than $80^{\circ}$ the rim emission is likely absorbed by the outer regions of the disk and the IN05 model can not be applied.

In practice, we compute visibility models for each object varying $a, \iota$ and PA independently. We then select the best models calculating the reduced $\chi^{2}$ between the visibility data and the theoretical values calculated at the same points in the $u-v$ plane. The observed near-infrared fluxes, and the IOTA data when available, are then "a posteriori" used to check the quality of the fit and, when possible, to reduce the degeneracy due to the small number of visibility points. For some stars, the existing data do not constrain the parameters, but still define a range, outside of which the fit to the data is very poor.

Table 2 shows in Col. 2 the best values of the astronomical silicate radius $a$ and, in Col. 3, the corresponding values of the radius of the inner $\operatorname{rim} R_{\text {rim }}$. The two observational parameters $i$ and PA are given in Cols. 4 and 5, respectively. Note that the free parameters are in boldface; $R_{\text {rim }}$ is a derived quantity.

\section{2. $M W C 758$}

PTI visibilities are fitted by a family of models, with parameters varying between the two extreme cases shown in Fig. 3. In one case, the disk has small grains of radius $a=0.17 \mu \mathrm{m}, \iota=48^{\circ}$ and $\mathrm{PA}=134^{\circ}$; in the other, big grains with $a \geq 1.2 \mu \mathrm{m}, \iota=40^{\circ}$ and $\mathrm{PA}=145^{\circ}$. Models with $a$ values within this range will fit the observed visibilities equally well, provided that we vary $\iota$ and PA in an appropriately way.

However, if we consider also the constraints set by the SED at near-infrared wavelengths, we find that only models with big grains fit it reasonably well (see the right panel of Fig. 3). The best-fitting model $\left(\chi_{r}^{2}=2.0\right)$ has then $a \geq 1.2 \mu \mathrm{m}$, inner rim radius is $R_{\text {rim }}=0.32 \mathrm{AU}$, rim effective temperature (at $z=0$ ) is $1460 \mathrm{~K}$. The near-infrared flux, $L_{\mathrm{NIR}}$, integrated between $2 \mu \mathrm{m}$ and $7 \mu \mathrm{m}$ is $25 \%$ of the total stellar luminosity, similar to the observed value. Once we fix $a$, the formal uncertainties on $\iota$, estimated from the surface where the reduced $\chi^{2}$ equals $\chi_{\min }^{2}+1$, 

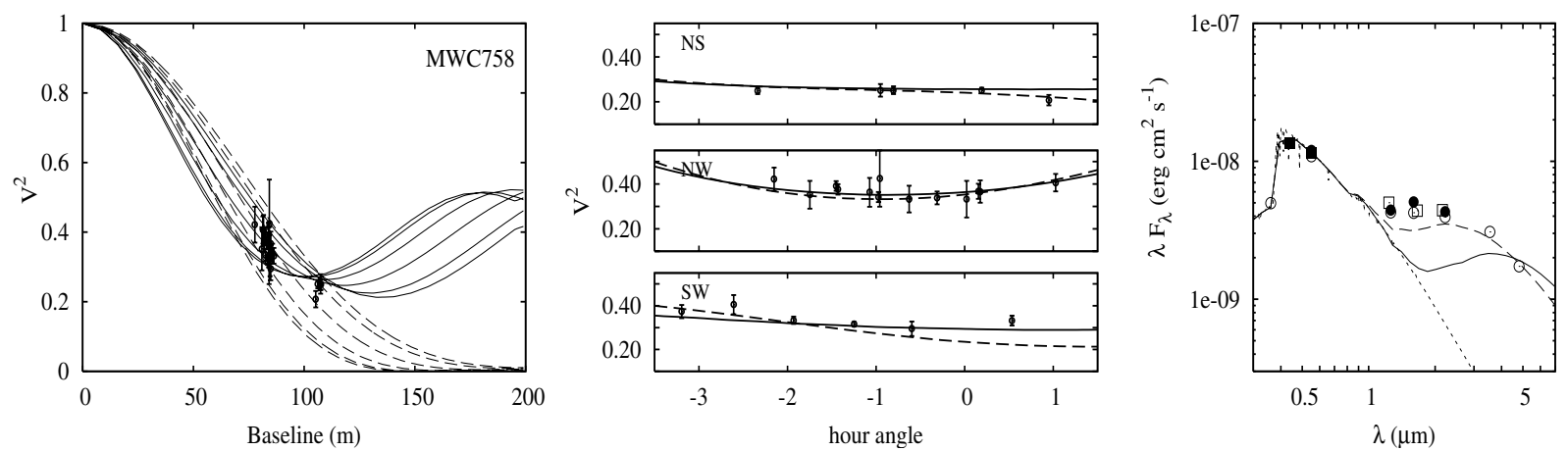

Fig. 3. The left and central panels show $K$-band $V^{2}$ data for MWC 758 respectively plotted as function of the baseline and of the hour angle, for the three different PTI baseline orientations (NS, baseline legth of $110 \mathrm{~m}$ in direction North-South; NW, $86 \mathrm{~m}$ direction North-West; SW, $87 \mathrm{~m}$ direction South-West). PTI measurements (Eisner et al. 2004) are shown by dots. The right panel shows the spectral energy distribution of MWC 758. The de-reddened photometric fluxes are from Eisner et al. (2004, filled circles), Malfait et al. (1998, empty circles), van den Ancker et al. (1998, filled squares) and Cutri et al. (2003, empty squares); the dotted line shows the photospheric stellar flux. The solid lines show the predictions of the best-fitting model with small grains $\left(a=0.17 \mu \mathrm{m}, \iota=48^{\circ}, \mathrm{PA}=134^{\circ}\right)$; the dashed lines the best-fitting model with big grains $\left(a \geq 1.2 \mu \mathrm{m}, \iota=40^{\circ}, \mathrm{PA}=145^{\circ}\right)$. As described in Sect. 4.2, all the intermediate disk configuration can reproduce the observations at almost the same level of confidence. Each curve on the left panel corresponds to a different orientation of the baseline on the plane of the sky (see Fig. 2). The stellar parameters are given in Table 1 .
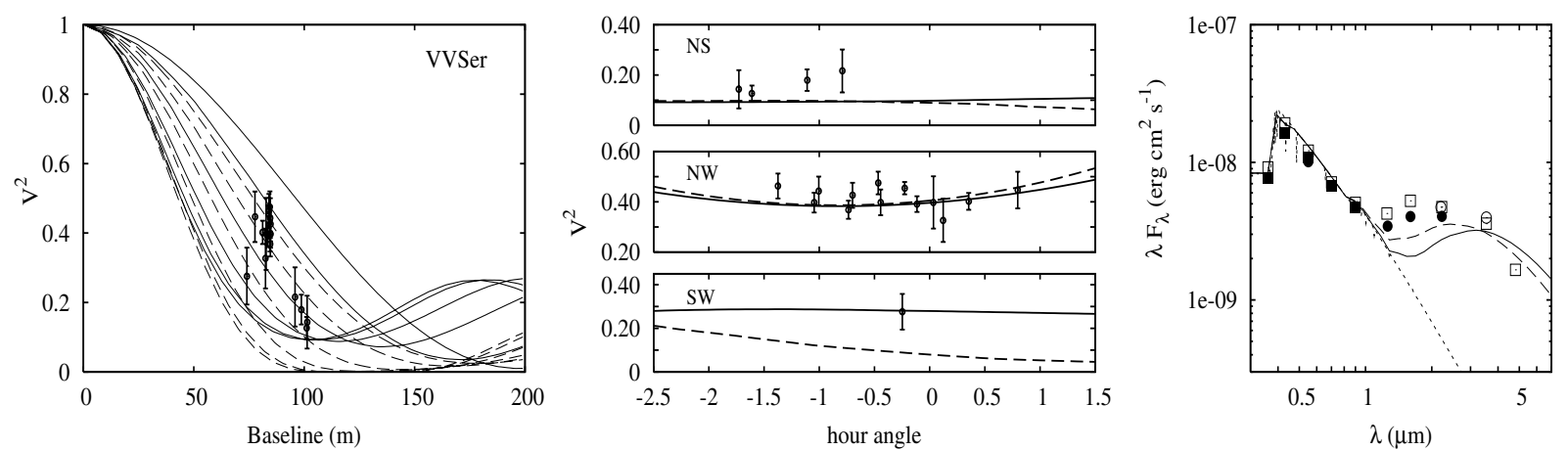

Fig. 4. Same as Fig. 3 for VV Ser. The solid curves show the model predicted values of the $K$-band $V^{2}$ for a rim with $a=0.52 \mu \mathrm{m}, \iota=65^{\circ}$ and $\mathrm{PA}=80^{\circ}$; the dashed curves for a rim model with $a \geq 1.2 \mu \mathrm{m}, \iota=55^{\circ}$ and PA $=115^{\circ}$. The photometric data are from Eisner et al. (2004, filled circles), Cutri et al. (2003, empty squares), Hillenbrand et al. (1992, empty squares), Rostopchina et al. (2001, filled squares). Stellar parameters in Table 1 .

are quite small, $\pm 3^{\circ}$. More realistic uncertainties are of the order of $10^{\circ}$ for both $\iota$ and PA.

Note that in MWC 758 the PTI visibilities define quite well the orientation of the disk, even when the SED is not used to constrain the grain size. In particular, the inclination cannot be lower than about $30^{\circ}$.

\subsection{VV Ser}

The results for the star VV Ser are shown in Fig. 4. As for MWC 758, the interferometric observations allow different sets of model parameters. Namely, we obtain similar values of the reduced $\chi^{2}(\sim 1.2)$ for all grain sizes $a \gtrsim 0.4 \mu \mathrm{m}$. Over this range of $a$, inclination and position angle vary in the intervals $45^{\circ}-80^{\circ}$ and $60^{\circ}-120^{\circ}$, respectively, with lower inclinations for larger grains. The correlation between $\iota$ and PA is very strong, and the uncertainties in these two parameters remain very large even for fixed $a$.

Although the fit is never very good, the VV Ser SED is better accounted for by large grains (see the right panel of Fig. 4) and in Table 2 we show the best values of the parameters for $a \geq 1.2 \mu \mathrm{m}$. The rim effective temperature is $1400 \mathrm{~K}$, the nearinfrared excess is $21 \%$ of $L_{\star}$.

\subsection{CQ Tau}

The limited number of visibility points does not allow us to constrain all the parameters of the disk. Figure 5 shows two models, with the same level of confidence $\left(\chi_{r}^{2} \sim 1\right)$; the two models have similar orientations (inclinations of $52^{\circ}$ and $46^{\circ}$ with position angles of $168^{\circ}$ and $164^{\circ}$ respectively) but very different grain sizes ( $a=0.3 \mu \mathrm{m}$ and $a \geq 1.2 \mu \mathrm{m})$ and radii of the inner rim $(0.25 \mathrm{AU}$ and $0.16 \mathrm{AU}$, respectively). For $a \geq 1.2 \mu \mathrm{m}$, the effective temperature of the rim (at $z=0$ ) is $T_{\text {eff }}=1480 \mathrm{~K}$, while $T_{\text {eff }}=1050$ for $a=0.3 \mu \mathrm{m}$. The SEDs of the two models are compatible with the observed fluxes, with $L_{\mathrm{NIR}}=13 \%-18 \% L_{\star}$. All the models with $a$ within this range, and similar $\iota$ and PA, have similar $\chi^{2}$ values. Outside this range, models give a much poorer fit to the data.

As for MWC 758, the visibility data constrain well the orientation of the disk on the sky. The inclination, in particular, has to be quite large, $40^{\circ} \lesssim \iota \lesssim 55^{\circ}$.

\section{5. $V 1295 \mathrm{Aql}$}

The PTI observations of V1295 Aql are characterized by a very small number of visibility points and the disk parameters are hardly constrained. Even adding the IOTA data does not help due to the big errors that affect these observations. Note also that PTI 

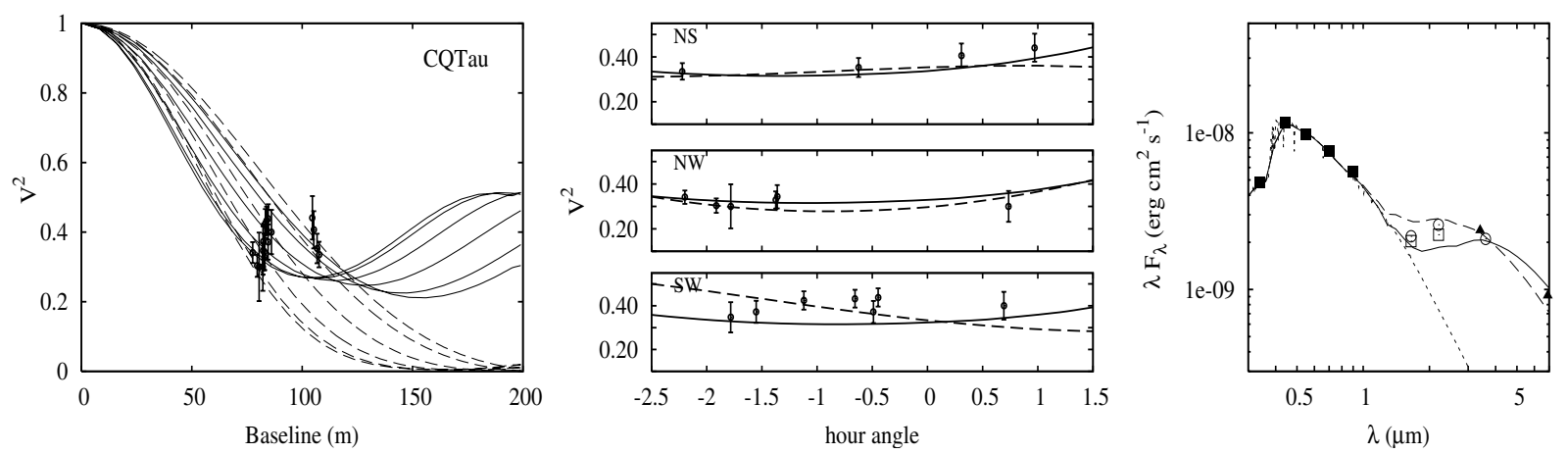

Fig. 5. Same as Fig. 3 for CQ Tau. The solid curves show the predictions of the model with $a=0.3 \mu \mathrm{m}\left(R_{\text {rim }}=0.25 \mathrm{AU}\right), \iota=52^{\circ}$ and PA $=168^{\circ}$; the dashed curves the model with $a \geq 1.2 \mu \mathrm{m}\left(R_{\text {rim }}=0.16 \mathrm{AU}\right), \iota=46^{\circ}$ and PA $=164^{\circ}$. The photometric data are from Eisner et al. (2004, empty squared), Glass \& Penston (1974, filled squares), ISO catalogue (empty circles) and Natta et al. (2001, filled triangles) and references therein.
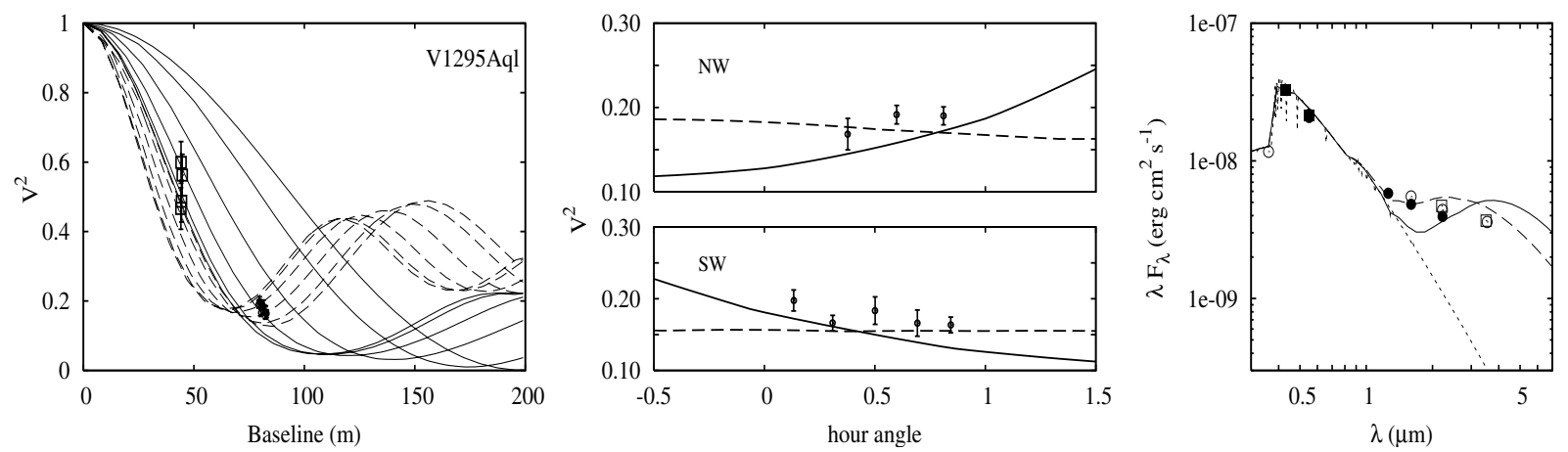

Fig. 6. Same as Fig. 3 for V1295 Aql. The solid lines plots the results of a model with $a \geq 1.2 \mu \mathrm{m}\left(R_{\text {rim }}=0.7 \mathrm{AU}\right), \iota=63^{\circ}$ and PA $=162^{\circ}$. The dashed lines a model with $a=0.3 \mu \mathrm{m}\left(R_{\mathrm{rim}}=1.2 \mathrm{AU}\right), \iota=40^{\circ}$ and PA $=80^{\circ}$. The photometric measures are from Eisner et al. (2004, filled circles), Malfait et al. (1998, open circles), Kilkenny et al. (1985, filled squared) and Glass \& Penston (1974, open squared). The short baseline points in the left panel (empty squares) are from IOTA, while the central panel shows only the PTI data.

and IOTA observations are performed at different wavelengths, $K$ and $H$ respectively.

As for CQ Tau, we show the models for the two extreme sets of parameters that give an equally good fit (Fig. 6) with $\chi_{r}^{2} \sim 1$. All the intermediate combinations of $a, \iota$ and PA can explain the observations as well. In order to fit the values of visibility, an inclination ranging between $40^{\circ}$ and $65^{\circ}$ is required. More faceon systems can not in general reproduce the visibility spread in the IOTA data and the $V^{2}-H A$ behaviour of the PTI points. The grain radius varies from $a \geq 1.2 \mu \mathrm{m}\left(R_{\text {rim }}=0.7 \mathrm{AU}, T_{\text {eff }}=\right.$ $1400 \mathrm{~K})$ to $a=0.3 \mu \mathrm{m}\left(R_{\text {rim }}=1.2 \mathrm{AU}, T_{\text {eff }}=970 \mathrm{~K}\right)$ while the position angle can not be defined at all.

The right panel of Fig. 6 shows the comparison between the observed and the predicted SED. More inclined disks can in general reproduce better the photometric measurements around $1.5 \mu \mathrm{m}$. The near-infrared emission of disk with an inclination of less than $50^{\circ}$ is peaked at about $4 \mu \mathrm{m}$ and can not reproduce the infrared excess between $1 \mu \mathrm{m}$ and $2 \mu \mathrm{m}$; in both models, the integrated near-infrared flux is $L_{\mathrm{NIR}} \sim 20 \% L_{\star}$.

\section{6. $M W C 480$}

Also in this case, due to the narrow range of available baselines, the PTI visibilities of MWC 480 are consistent with different sets of parameters at the same level of confidence $\left(\chi_{r}^{2} \sim 2\right)$. Figure 7 shows the two extreme disk configurations characterized by quite similar parameters for the dust grain size $(a=0.2-0.3 \mu \mathrm{m}$; $R_{\text {rim }}=0.63-0.53 \mathrm{AU}$ and $\left.T_{\text {eff }} \simeq 1250 \mathrm{~K}\right)$, but very different values of the inclination $\left(\iota=35^{\circ}\right.$ and $\left.\iota=60^{\circ}\right)$ and the position angle $\left(\mathrm{PA}=60^{\circ}\right.$ and $\left.\mathrm{PA}=168^{\circ}\right)$. Several intermediate configurations reproduce the observed data as well. The degeneracy can not be removed even using the SED (Fig. 7) which is similar in the two models $\left(L_{\mathrm{NIR}} / L_{\star}=0.18-0.14\right)$ and it is only roughly consistent with the photometric values.

\section{7. $A B$ Aur}

AB Aur is the only HAe star that cannot be fitted with the IN05 models. The PTI visibilities require a face-on rim, consistent with the inclination derived from large-scale images in scattered light (Grady et al. 1999; Fukagawa et al. 2004) and at millimeter wavelengths (Corder et al. 2005; Piétu et al. 2005). For these inclinations, the $V^{2}$ data imply a very small inner radius, about two times smaller that the smallest $R_{\text {rim }}$ obtained using the IN05 model (see Fig. 8). If, to put the discrepancy in a more physical context, we take $T_{\text {evp }}$ as a free parameter, we find good agreement with the PTI data for $T_{\text {evp }} \sim 2800 \mathrm{~K}$ (dashed line), a value by far too high not only for silicates but also for any other type of grains (e.g., Pollack et al. 1994).

The situation becames even less clear if we consider also the IOTA observations (squared points), since they seem to indicate the presence of a more inclined disk with an inner radius between $0.26 \mathrm{AU}$ and $0.51 \mathrm{AU}$. No additional information can be obtained from the analysis of the spectral energy distribution, since all the inner rim models with an effective temperature between $1500 \mathrm{~K}$ and $2500 \mathrm{~K}$ are compatible with the photometric data. We will come back to AB Aur in Sect. 6. 

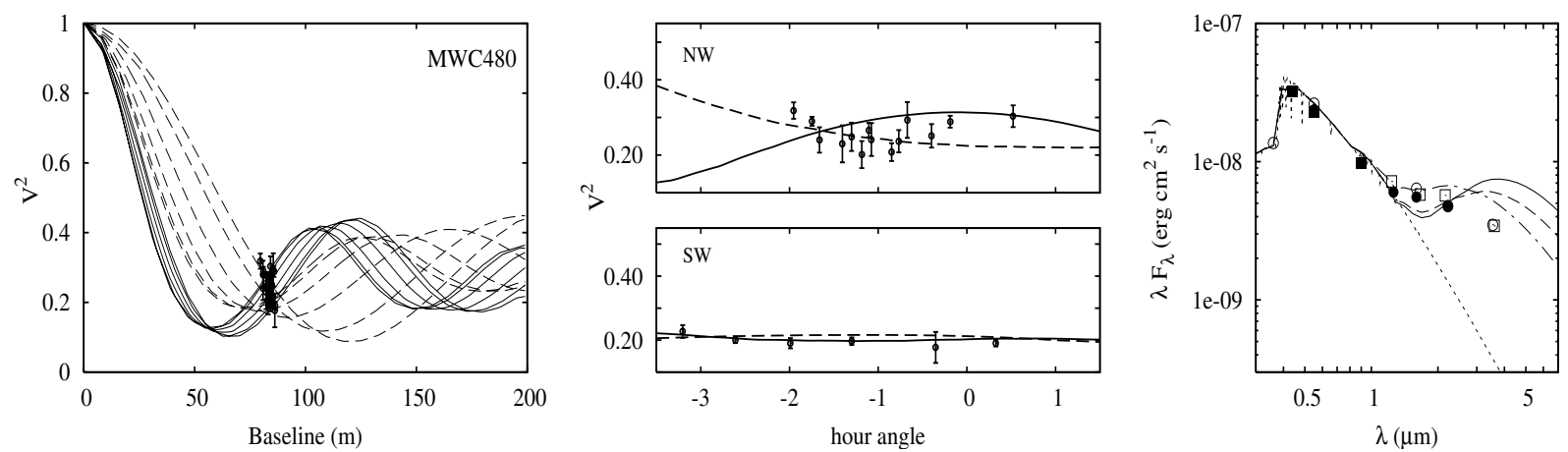

Fig. 7. Same as Fig. 3 for MWC 480. The solid lines are for the disk model characterized by $a=0.2 \mu \mathrm{m}\left(R_{\text {rim }}=0.63 \mathrm{AU}\right), \iota=35^{\circ}$ and PA $=30^{\circ}$. The dashed lines are relative to a disk model characterized by $a=0.3 \mu \mathrm{m}\left(R_{\mathrm{rim}}=0.53\right), \iota=60^{\circ}$ and PA $=102^{\circ}$. The photometric data are from Eisner et al. (2004, filled circles), Malfait et al. (1998, open circles), Cutri et al. (2003, empty squares) and van den Ancker et al. (1998, filled squares).

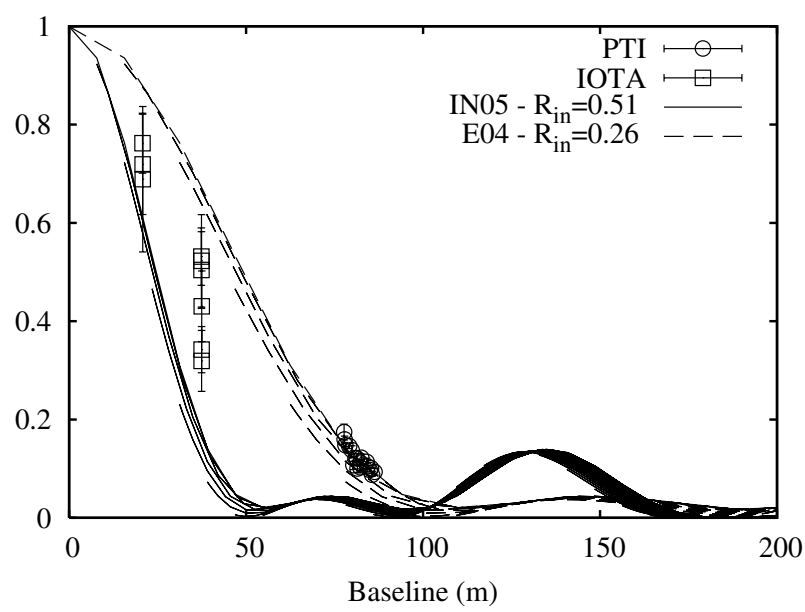

Fig. 8. $V^{2}$ data for $\mathrm{AB}$ Aur plotted in function of the baseline. The square and circle points refer respectively to the IOTA and PTI observations in $K$ band. The solid line is relative to the best prediction of the self-consistent model of inner $\operatorname{rim}\left(a \geq 1.2 \mu \mathrm{m}, R_{\text {rim }}=0.51 \mathrm{AU}\right.$ $\left.\iota=20^{\circ}\right)$. The dashed line is obtained with the IN05 model using $a \geq 1.2 \mu \mathrm{m}$ with an ad hoc $T_{\text {evp }}=2800 \mathrm{~K}\left(R_{\text {rim }}=0.26 \mathrm{AU}\right)$.

\section{Comparison with previous analysis}

Fits to the same interferometric data analyzed in Sect. 4 have been obtained by Eisner et al. (2004, hereafter E04) assuming a toroidal shape for the inner "puffed-up" rim, based on the simplified DDN01 model. In these fits, the free parameters are the location of the rim $R_{\text {rim }}$ and the two observational parameters, $\iota$ and PA. The E04 results are shown in Table 2.

We note that for three objects (MWC 758, VV Ser and CQ Tau) the E04 inclinations are in agreement within the errors with the values obtained with the IN05 model, while for the other two (V1295 Aql and MWC 480) the E04 ८ estimates are consistent with the lowest value of the range derived in this paper.

The largest differences are in the derived values of $R_{\text {rim }}$ : the IN05 inner radii are always larger than E04 results, with a maximum difference of a factor $\sim 3$ if we consider our maximum $R_{\text {rim }}$ in the MWC 480 system. While for CQ Tau the two values are almost the same, for all the other stars the difference is a factor 1.5 and 2. This discrepancy is mainly due to the difference between IN05 and E04 models. In particular, in IN05, the curved shape of the emitting surface is self-consistently calculated, allowing a more correct determination of the dependence of the rim emission on the inclination of the disk.

Moreover, the IN05 model takes into account the effect of the radiation transport within the disk, even if in an approximate way (see Appendix A in IN05). This supplementary heating is neglected by E04, who calculate the dust temperature taking into account only the direct stellar radiation. The ratio between the two values of $R_{\text {rim }}$ is given by the relation

$\frac{R_{\text {rim }}}{\hat{R}_{\text {rim }}}=\sqrt{\hat{\epsilon}(2+1 / \epsilon)}$,

where the $\hat{\epsilon}, \hat{R}_{\text {rim }}$ and $\hat{T}_{\text {evp }}^{2}$ are the values used by E04 and the inner radius $\hat{R}_{\text {rim }}$ is given by the relation

$\hat{R}_{\text {rim }}=\frac{1}{\hat{T}_{\text {evp }}^{2}} \sqrt{\frac{L_{\star}}{4 \pi \sigma} \frac{1}{\hat{\epsilon}}}$.

Assuming the same value of the dust emissivity $(\epsilon=\hat{\epsilon})$, the ratio $R_{\text {rim }} / \hat{R}_{\text {in }}$ is $\sim 1$ for $\epsilon \ll 1$. The difference increases for larger $\epsilon$ and is maximum when $\epsilon$ and $\hat{\epsilon}$ are very different.

Finally, there also differences due to the fact that E04 assumes $T_{\text {evp }}$ (or, equivalently, $R_{\text {rim }}$ ) as a free parameter, while in the IN05 model $T_{\text {evp }}$ is self-consistently determined starting from the choice of the type of grains and the gas density in the disk (see Eq. (2)). For all our target stars, the resulting values of $T_{\text {evp }}$ vary between $1370 \mathrm{~K}$ and $1460 \mathrm{~K}$, and are in some cases significantly different from those given by E04.

\section{Discussion}

The results presented in Sect. 4 show that, with the exception of AB Aur, the IN05 self-consistent models of the "puffed-up" inner rim can explain the available observations, both visibilities and SEDs, of HAe stars. They can be used to derive information about the properties of the dust present in the innermost region of the circumstellar disk, the location of the inner rim and the orientation of the disk on the sky, using a minimum number of assumptions.

\subsection{Presence of large grains}

As shown in Sect. 4, the IN05 models reproduce the interferometric data under the assumption that the most refractory dust 
in the inner disk is made of silicates, with properties typical of astronomical silicates (Weingartner \& Draine 2001).

In four cases, grain sizes larger than $\sim 1.2 \mu \mathrm{m}$ are either required by or consistent with the observations. Only in one case are the data better fitted with $a \sim 0.2-0.3 \mu \mathrm{m}$. Grains in the rim are thus larger, and often much larger, than grains in the interstellar medium ( $a=0.01-0.1 \mu \mathrm{m}$, Weingartner \& Draine 2001), confirming that grain growth has taken place in the innermost disk regions (van Boekel et al. 2004).

Even if the predicted near-infrared excess agrees well with the photometric observations, some interesting differences exist between the theoretical and the observed spectral energy distributions. With the exception of CQTau, the predicted SEDs always peak at a wavelength slightly longer than found in the observations: the flux at short wavelengths (between $1.5 \mu \mathrm{m}$ and $2.2 \mu \mathrm{m}$ ) is thus generally underestimated while the flux between $2.2 \mu \mathrm{m}$ and $7 \mu \mathrm{m}$ is overestimated. This may be due to the fact that in our models the SED is computed assuming that each point on the surface of the rim emits as a black body at the local effective temperature. This approximation is energetically correct but may not reproduce the exact wavelength dependence of the emitted radiation (see Appendix in IN05).

The rim models fail only in the case of AB Aur, where silicates, of whatever size, produce rims that are too distant from the star to be consistent with the observations. As shown in Sect. 4.7, PTI and IOTA data give somewhat contradictory results, and more interferometric data are clearly required. However, unless further observations drastically change the present picture, the discrepancy between the rim model predictions and the data is highly significant, and some of the basic underlying assumptions need to be changed. It is possible that in AB Aur grains more refractory than silicates dominate the dust population in the inner disk; however, the $T_{\text {evp }}$ required $(\sim 2800 \mathrm{~K})$ is too high for any dust species likely present in disks (e.g., Pollack et al. 1994).

It is more likely that gas in the dust-depleted inner region absorbs a significant fraction of the stellar radiation, shielding the dust grains which are therefore cooler than in our models. This requires a high gas density in the inner disk, as expected if the accretion rate is high; in general, the accretion rates of HAe stars (including $\mathrm{AB}$ Aur) are low enough to ensure that the gaseous disk remains optically thin (Muzerolle et al. 2004). However, our knowledge of the accretion properties and gas disks of HAe stars is very poor, and should be investigated further.

$\mathrm{AB}$ Aur may be more than just an oddity. The presence of optically thick gas inside the inner rim has been proposed to explain the near-infrared interferometric observations of some very bright Herbig Be stars, for which the visibility data suggest inner disk radii many times too small to be consistent with the "puffedup" rim models (Malbet et al. 2005; Monnier et al. 2005). If this is the case, AB Aur could be the low-luminosity tail of the same phenomenon, which, given its small distance and large brightness, could be used to understand a whole class of objects.

\subsection{Inclination and position angle}

Inclination and PA are well constrained by the existing data only in one case (MWC 758). We want to stress, however, that values of the parameters outside the ranges given in Table 2 do not fit the data at all. In particular, there are no objects consistent with face-on disks, or in general with a centro-symmetric brightness distribution. This rules out models, such as those of Vinkovic et al. (2005), where most of the near-infrared flux is contributed by a spherically symmetric shell around the star, rather than by a circumstellar disk.
Two stars (CQ Tau and VV Ser) belong to the group of UXOR variables, which are interpreted as objects with disks seen close to edge-on (Grinin et al. 2001; Natta \& Whitney 2001; Dullemond et al. 2003). We derive for them large inclinations, in agreement with this interpretation.

For some of our targets, there are in the literature estimates of the orientation of the outer disk on the plane of the sky obtained with millimeter interferometers (Testi el al. 2001, 2003; Manning \& Sargent 1997; Piétu et al. 2005; Corder et al. 2005). These determinations refer to the outer disk, i.e., to spatial scales of 50-100 AU at least. The comparison with the values derived in the near-infrared for the inner disk (on scales of less than $1 \mathrm{AU}$ ) can provide information on possible distortions in the disk, e.g. variations of the inclination with radius. For the three disks with millimeter data (MWC 758, CQ Tau and MWC 480), there is agreement (within the uncertainties) between the inclination obtained by infrared and millimeter observations However, it is certainly premature to exclude the existence of disk distortions, given the large uncertainties that affect both the millimeter and the near-infrared estimates. More accurate interferometric observations in the two wavelength ranges and self-consistent models of the disk at all physical scales are required.

An interesting case is that of VV Ser, whose disk has recently been imaged as a shadow seen against the background emission in the 11.3 PAH feature (Pontoppidan et al. 2006); These authors derive an inclination (of the outer disk) of about $70^{\circ}$ and a position angle of $13^{\circ} \pm 5^{\circ}$. While the inclination is consistent with the upper limit of the range we obtain for the inner disk, the position angle is off by almost $90^{\circ}$. This discrepancy is intriguing, and deserves further investigation.

\subsection{Improving the model constrains}

Near-infrared interferometric observations of disks around premain sequence stars are still few and sparse. It is clear from our analysis that even in the most favorable cases more visibility data at different baselines are necessary to narrow the range of possible disk inclinations and grain properties.

Given the huge demands of telescope time that interferometric observations require, it is useful to make use of model predictions in preparing the observations and in choosing the baseline configurations that can constrain the disk structure.

CQ Tau represents an example of how the IN05 model can be used in this context. To better constrain the inner rim structure, one will need observations with baselines longer than $130 \mathrm{~m}$ (available with the VLT interferometer), for which the predicted values of the squared visibility parameters are very different for different disk models (see Fig. 5). On the other hand, observations with baseline shorter than $60 \mathrm{~m}$ could better constrain the inner radius of MWC 480, since a degeneracy in the models is present at longer baselines.

V1295 Aql represents a still different case, in which the degeneracy in the values of the predicted squared visibility can be removed observing at distant hour angles for the same baseline configurations, in order to determine the visibility variations at the same baseline, due to the inclination of the disk.

\section{Summary and conclusions}

In this paper we have analyzed the near-infrared interferometric observations of the six best observed HAe stars using the rim models developed by Isella \& Natta (2005). Our aim was to explore the potential of near-infrared interferometry to constrain the properties of the grains in the inner disks of these stars. 
The basic assumptions of the IN05 rim models are that the inner disk structure is controlled by the evaporation of dust in the unattenuated stellar radiation field, as expected if the gaseous disks have low optical depth, and that the most refractory grains are silicates. The IN05 self-consistent models for the "puffedup" inner rim reproduce both the interferometric observations and the near-infrared spectral energy distribution of all the objects we have studied, with the exception of $A B$ Aur, which we have briefly discussed.

For the five stars where we are able to obtain a good fit to the data, we can estimate the grain sizes in the rim, i.e., in the midplane of the inner disk. We find that in four cases grains larger than $\sim 1.2 \mu \mathrm{m}$ are either required by or consistent with the data. Only in one case do we find that the existing data require $a \sim 0.2-0.3 \mu \mathrm{m}$. Note that this value of $a=1.2 \mu \mathrm{m}$ is a lower limit to the grain size: grains can be much larger, since the rim location and shape do not change significantly if the grains grow further.

As a result of the model-fitting, one derives also the inclination and position angle of the disk on the plane of the sky. We find that, in general, these parameters are not well constrained by the existing data. However, in all cases we can fit, inclinations lower than $30^{\circ}$ are not consistent with the observations and the surface brightness distribution can not be circularly symmetric. This rules out a spherical envelope as the dominant source of the near-infrared emission.

For some objects, estimates of the inclination of the outer disk have been obtained from millimeter interferometric observations; within the uncertainties, they agree with the values obtained for the inner disk.

Our analysis shows that near-infrared interferometry is a very powerful tool for understanding the properties of the inner disks, in particular when combined with physical models of these regions. However, at present the existing data are for many objects still too sparse in their coverage of the $u-v$ plane to allow an accurate determination of the disk parameters. We expect that this will be improved in the future. In this context, since nearinfrared interferometry is and will remain a very time demanding technique, we stress the importance of using physical models of the inner region of the disk in planning future observations.

\section{References}

Calvet, N., Patiño, A., Magris, G. C., \& D'Alessio, P. 1991, ApJ, 380, 617C

Calvet, N., Magris, G. C., Patiño, A., \& D’Alessio, P. 1992, Rev. Mex. Astron. Astrofis., 24,

Chiang, E. I., \& Goldreich, P. 1997, ApJ, 490, 368

Chiang, E. I., Joung, M. K., Creech-Eakman, M. J., et al. 2001, ApJ, 547, 1077

Colavita, M., Akeson, R., Wizinowich, P., et al. 2003, ApJ, 592, 83

Corder, S., Eisner, J., \& Sargent, A. I. 2005, ApJ, 622L, 133C

Cutri, R. M., Skrutskie, M. F., van Dyk, S., et al. 2003, yCat, 2246, 0C

Draine, B. T. 1985, ApJS, 57, 587D

Dominik, C., Dullemond, C. P., Waters, L. B. F. M., \& Walch, S. 2003, A\&A, 398, 607

Dullemond, C. P. 2000, A\&A, 361, L17

Dullemond, C. P., Dominik, C., \& Natta, A. 2001, ApJ, 560, 957

Dullemond, C. P., van den Ancker, M. E., Acke, B., \& van Boekel, R. 2003, ApJ, 594, 47 Dullemond, C. P., \& Dominik, C. 2004, A\&A, 417, 159

Eisner, J. A., Lane, B. F., Akeson, R. L., Hillebrand, L. A., \& Sargent, A. I. 2003, ApJ, 588, 360

Eisner, J. A., Lane, B. F., Hillebrand, L. A., et al. 2004, ApJ, 613, 1049

Eisner, J. A., Hillebrand, L. A., White, R. J., Akeson, R. L., \& Sargent, A. I. 2005 [arXiv: astro-ph/0501308]

Fukagawa, Hayashi, M., Tamura, M., et al. 2004, ApJ, 605, 53

Glass, I. S., \& Penston, M. V. 1974, MNRAS, 167, 237G

Grady, C. A., Woodgate, B., Bruhweiler, F. C., et al. 1999, ApJ, 523, L151

Grady, C. A. 2000, ApJ, 544, 859

Grinin, V. P., Kozlova, O. V., Natta, A., et al. 2001, A\&A, 379, 482

Hillenbrand, L. A., Strom, S. E., Vrba, F. J., \& Keene, J. 1992, ApJ, 397, 613

Kilkenny, D., Whittet, D. C. B., Davies, J. K., et al. 1985, SAAOC, 9, 55K

Isella, A., \& Natta, A. 2005, A\&A, 438, 899I

Malbet, F., Benisty, M., De Wit, W. J., et al. 2005 [arXiv: astro-ph/0510350]

Malfait, K., Boegaert, E., \& Waelkens, C. 1998, A\&A, 331, 211

Mannings, V., \& Sargent, A. I. 1997, ApJ, 490, 792

Meeus, G., Sterzik, M., Bouwman, J., \& Natta, A. 2003, A\&A, 409L, 25M

Millan-Gabet, R., Schloerb, P. F., \& Traub, W. A. 2001, ApJ, 546, 358

Monnier, J. D., \& Millan-Gabet, R. 2002, ApJ, 597, 694

Monnier, J. D., Millan-Gabet, R., Billmeier, R., et al. 2005, ApJ, 624, 832M

Muzerolle, J., D’Alessio, P., Calvet, N., \& Hartmann, L. 2004, ApJ, 617, 406

Natta, A., \& Whitney, B. A. 2000, A\&A, 364, 633

Natta, A., Prusti, T., Neri, R., Grinin, V. P., \& Mannings, V. 2001, A\&A, 371, 186

Natta, A., Testi, L., Calvet, N., et al. 2006, PPV proceeding

Piétu, V., Guilloteau, S., \& Dutrey, A. 2005, A\&A, 443, 945

Pollack, J. B., Hollenbach, D., Beckwith, S., et al. 1994, ApJ, 421, 615

Pontoppidan, et al. 2006, preprint

Rostopchina, A. N., Grinin, V. P., \& Shakhovskoi, D. N. 2001, Arep, 45, 51

Simon, M., Dutrey, A., \& Guilloteau, S. 2000, ApJ, 545, 1034

Straizys, V., Cernis, K., \& Bartasiute, S. 1996, Baltic Astronomy, 5, 125

Testi, L., Natta, A., Shepherd, D. S., \& Wilner, D. J. 2001, A\&A, 554, 1087 T

Testi, L., Natta, A., Shepherd, D. S., \& Wilner, D. J. 2003, A\&A, 403, 323T

Tuthill, P. G., Monnier, J. D., \& Danchi, W. C. 2001, Nature, 409

van Boekel, R., Waters, L. B. F. M., Dominik, C., et al. 2003, A\&A, 400, L21

van Boekel, R., Min, M., Leinert, Ch., et al. 2004, Nature, 432, 479

van den Ancker, M. E., de Winter, D., \& Tjin A Djie, H. R. E. 1998, A\&A, 330, 145V

Vinkovic, D., Ivezic, Z., Tomislav, J., \& Moshe, E. 2005 [arXiv: astro-ph/0506154]

Weingartner, J. C., \& Draine, B. T. 2001, ApJ, 548, 296 